Ph. Getto · O. Diekmann · A.M. de Roos

\title{
On the (dis) advantages of cannibalism
}

Received: 17 June 2003 / Revised version: 5 July 2005 /

Published online: 10 November 2005 - (C) Springer-Verlag 2005

Abstract. Cannibalism is an interaction between individuals that can produce counterintuitive effects at the population level. A striking effect is that a population may persist under food conditions such that the non-cannibalistic variant is doomed to go extinct. This so-called life boat mechanism has received considerable attention. Implicitly, such studies sometimes suggest, that the life boat mechanism procures an evolutionary advantage to the cannibalistic trait.

Here we compare, in the context of a size structured population model, the conditions under which the life boat mechanism works, with those that guarantee, that a cannibalistic mutant can invade successfully under the steady environmental conditions as set by a non-cannibalistic resident. We find qualitative agreement and quantitative difference. In particular, we find that a prerequisite for the life boat mechanism is, that cannibalistic mutants are successful invaders. Roughly speaking, our results show that cannibalism brings advantages to both the individuals and the population when adult food is limiting.

\section{Introduction}

Cannibalism is an intriguing phenomenon as it seems, almost literally, like cutting into ones own flesh. The aim of this paper is, to contribute to the weighing of advantages against disadvantages, with due attention for the distinction between the individual level and the population level.

To set the scene, we first give an incomplete list of relevant aspects, while referring to the survey article (Polis 1981) and to our concluding section for a more complete and detailed list and especially to (Polis 1981) for numerous references.

By practicing cannibalism, an individual gains energy (perhaps in the precious form of a scarce resource, like protein). Moreover, it eliminates a (potential, future) rival (for mates), competitor (for food) and aggressor (of offspring).

Ph. Getto: Department of Mathematics, University of Utrecht, P.O. Box 80010, 3580 TA Utrecht, The Netherlands

current address: Institute of Applied Mathematics and Mechanics, Faculty of Mathematics, Informatics and Mechanics, Warsaw University, ul. Banacha 2, 02-097 Warszawa, Poland

O. Diekmann: Department of Mathematics, University of Utrecht, P.O. Box 80010, 3580 TA Utrecht, The Netherlands

A.M. de Roos: Institute for Biodiversity and Ecosystem Dynamics, University of Amsterdam, P.O. Box 94084, 1090 GB Amsterdam, The Netherlands

Correspondence to: $\mathrm{Ph}$. Getto, e-mail address: phgetto@yahoo.com

Key words or phrases: Cannibalism - Chemostat dynamics - Size structure - Steady states - Interaction variables - Nonlinear dynamical system - Adaptive dynamics 
As emphasized by BSE among cattle and Kuru among humans, (involuntary) cannibalism may strongly contribute to pathogen transmission. Moreover, it may lead to injuries (in particular, when the size difference between agressor and potential victim is not too big).

In this paper we ignore pathogens. Moreover, we take size differences between individuals into account and assume, that the size of the potential victim is so much smaller than that of the cannibal, that injuries of the cannibal may be neglected as well.

When parents and offspring live close together, a cannibalistic individual has a higher chance to consume its own offspring than that of an arbitrary individual. We ignore such a consequence of spatial structure and pretend that the world is well-mixed.

The (dis) advantages at the i-level resulting from cannibalism are as follows. First, a juvenile has of course a chance of being eaten by a conspecific adult, whereas an adult receives additional energy when cannibalizing a juvenile. But an adult also faces a possible disadvantage related to optimal foraging: perhaps the handling time and digestion capacity spent on intraspecific prey are better spent on other sources of food?

What is the effect at the population level resulting from these mechanisms? If positive aspects at the individual level do not play a role, one expects of course negative effects at the population level. An early and detailed example of such a negative effect is given in (Botsford 1981). In this paper cannibalism is modeled as a density dependent phenomenon and energy gain for the predator is not taken into account. It is analyzed how, at a low population level, the effect of population density on individual survival to recruitment (e.g. via cannibalistic predation) can, under the assumption that low population levels result in an increase in individual growth rate, contribute to the maintenance of a low population level. (In fact this cannibalism model is the first in which bistability is found).

As shown in several papers, however, see (Van den Bosch et al. 1988), (Henson 1995), (Van den Bosch and Gabriel 1997), (Diekmann 1999) and (Diekmann et al. 2003), when taking energy gain of the cannibal into account, the net effect at the p-level may be positive, in the sense that the population persists under food conditions such that, without cannibalism, the population would go extinct (the name "life boat mechanism" suggests, perhaps, that such food conditions occur only every now and then). The key point is that juveniles have access to food which is too small (or otherwise inaccessible) for the adults themselves. So, in a sense, the juveniles form part of the foraging strategy of the adults.

In all earlier work on the life boat mechanism that we are aware of, the juvenile food and the non-cannibalistic adult food were taken constant, i.e., not influenced by the population itself through consumption. Moreover, in many models juvenile food was not considered explicitly. In the present paper we distinguish between juvenile and adult food explicitly and incorporate that their steady state levels are influenced via consumption by the size and composition of the population. In loose terms our main conclusion is that the life boat mechanism manifests itself when the adult food is the limiting resource, while being irrelevant when the juvenile food is the limiting resource. 
Another main theme of this paper is, that from the point of view of evolution by natural selection, the life boat mechanism is not what counts. Indeed, as the theory of Adaptive Dynamics (Metz et al. 1992, 1996), (Dieckmann and Law 1996), (Geritz et al. 1997, 1998) and (Diekmann 2004) tells us, we should investigate whether a (slightly) cannibalistic small subpopulation starts to grow exponentially in the environmental (= food) conditions as set by the non-cannibalistic (so called "resident") population. Phrased this manner, the question concerns the evolutionary onset of cannibalism. After it has been answered, further questions arise naturally: what degree of cannibalism should we expect? What does, apart from physiological constraints due to size-size incompatibility, determine the size-window in which individuals are vulnerable to intraspecific predation by an individual of a given size? Etcetera. As we shall show, the "evolutionary onset" question is easier to answer than the "lifeboat" question: standard optimal foraging arguments suffice. The reason is that evolution is short sighted: only immediate gains and losses count and not how the world will be after the successful mutant has ousted the former resident.

Yet, fortunately, the two themes do not clash. In, once again, loose terms, the conclusion of the analysis in adaptive dynamics spirit is, that cannibalism will originate if adult food is the limiting resource, while it will not, if juvenile food is the limiting resource. So, despite the fact that the quantitative details of the "life boat" condition and the "evolutionary onset" condition differ substantially, we can say, that at a qualitative level the population advantages of cannibalism manifest themselves exactly when the individual advantages lead to cannibalism. When interpreted in a certain way, the life boat population phenomenon gives a clue concerning the evolutionary advantages of cannibalistic clans.

Exploiting the "only one state at birth" feature, we rewrite the steady state problem as a nonlinear system of equations in the so-called environmental interaction variable $I$ and the population birth rate $c$. The technical basis for the bifurcation analysis that we outline at the beginning of Section 4, is provided in more generality in Steady state analysis for a size structured cannibalism model with two dynamic resources, which is a chapter in the $\mathrm{PhD}$ thesis of the first author (Getto, thesis) and available on request. In the latter one finds an extension of the bifurcation analysis in (Diekmann et al. 2003) to an infinite-dimensional environmental interaction variable $I$, as appearing in the parametrization of (Claessen and de Roos 2002) or (Claessen 2002), and two dynamic resources.

The bifurcation parameter that we consider is the position along a curve in the two parameter plane of the carrying capacities of, respectively, juvenile and adult food.

\section{The model formulation}

In an attempt to bring out the bare essentials, we sacrifice much of the generality of the model proposed in (Getto, thesis). In particular we assume here that juveniles, and only juveniles, grow and that adults, and only adults, reproduce. Only adults cannibalise and they can only cannibalise juveniles of suitable sizes. A generality we allow for, however, is the existence of two dynamic standard food sources, one 
for juveniles and one for adults. So juveniles compete for food among themselves and so do adults. But adults that are cannibalistic do have an additional source of food: the juveniles.

We assume that every individual is born with size $x_{b}$ and turns into an adult upon reaching size $x_{A}$. Juveniles of size $x$ experience a force of mortality $\mu_{1}(x)$ and, possibly, an additional force of mortality due to cannibalism. Adults experience a force of mortality $\mu_{2}$.

We now specify our assumptions on individual growth, reproduction and maintenance in terms of a partitioning of the individual's energy budget. More precisely, we use a so-called net-assimilation model, in contrast to a net-reproduction model, see (Kooijman 2000) for details (also for the case where growth and reproduction occur "simultaneously"). We denote by $Z_{1}$ the density/concentration of juvenile food and by $Z_{2}$ that of adult food. Incorporating a Holling Type II functional response, a juvenile individual of size $x$ gains energy at rate

$$
\frac{E_{1}(x) C_{1}(x) Z_{1}}{1+H_{1}(x) C_{1}(x) Z_{1}},
$$

where $C_{1}$ is the size-specific attack rate, $H_{1}$ the size-specific handling time (or, alternatively, a measure for the digestive capacity involved) and $E_{1}$ the amount of energy gained from a unit of food (which is allowed to be size-specific).

We assume that maintenance costs are proportional to size and that, in the case of juveniles, all remaining energy is scheduled to growth. Hence we have

$$
\begin{aligned}
\frac{d x}{d a} & =\eta_{1}\left(\frac{E_{1}(x) C_{1}(x) Z_{1}}{1+H_{1}(x) C_{1}(x) Z_{1}}-\zeta_{1} x\right), \\
x(0) & =x_{b}
\end{aligned}
$$

to describe how the size $x$ of a juvenile individual changes with its age $a$ (here $\eta_{1}$ takes care of converting energy units into size units and $\zeta_{1}$ is the amount of energy per unit of time and unit of size needed for maintenance). Implicitly we here assume that the energy ingested exceeds what is needed for maintenance, which is always true in a nontrivial equilibrium.

Analogously, a non-cannibalistic adult gains energy at rate

$$
\frac{E_{2} C_{2} Z_{2}}{1+H_{2} C_{2} Z_{2}}
$$

and, after paying for maintenance, uses this energy for reproduction. In other words, a non-cannibalistic adult produces offspring at rate

$$
\eta_{2}\left(\frac{E_{2} C_{2} Z_{2}}{1+H_{2} C_{2} Z_{2}}-\zeta_{2} x_{A}\right) .
$$

We assume that food is produced at a constant rate and that decay of food is a linear process. So in the absence of consumption the food density/concentration would 
stabilize at a certain level, which we denote by, respectively, $K_{1}$ (juvenile food) and $K_{2}$ (adult food). If we now include the effect of consumption we arrive at equations

$$
\begin{aligned}
& \frac{d Z_{1}}{d t}=r_{1}\left(K_{1}-Z_{1}\right)-\int_{x_{b}}^{x_{A}} \frac{C_{1}(x) Z_{1}}{1+H_{1}(x) C_{1}(x) Z_{1}} n(\cdot, x) d x, \\
& \frac{d Z_{2}}{d t}=r_{2}\left(K_{2}-Z_{2}\right)-\frac{C_{2} Z_{2}}{1+H_{2} C_{2} Z_{2}} N_{A}(\cdot)
\end{aligned}
$$

to describe the dynamics of the food concentrations. Here $n(t, \cdot)$ is the size-density of juveniles at time $t$ and $N_{A}(t)$ the density/number of adults at time $t$.

To incorporate the effects of cannibalism we adjust two model components: the juvenile death rate and the adult energy intake rate. The quantities $C, H$ and $E$ provided with an index $y$ refer to a victim of size $y$. When adults are cannibalistic we replace (2.3) by

$$
\frac{E_{2} C_{2} Z_{2}+\int_{x_{b}}^{x_{A}} E_{y} C_{y} n(\cdot, y) d y}{1+H_{2} C_{2} Z_{2}+\int_{x_{b}}^{x_{A}} H_{y} C_{y} n(\cdot, y) d y}
$$

and adapt (2.4) and (2.6) (the latter only in the denominator) accordingly, while putting the per capita death rate of juveniles of size $x$ equal to

$$
\mu_{1}(x)+\frac{C_{x} N_{A}(\cdot)}{1+H_{2} C_{2} Z_{2}+\int_{x_{b}}^{x_{A}} H_{y} C_{y} n(\cdot, y) d y} .
$$

\section{The steady state equations for a noncannibalistic population}

We first consider a non-cannibalistic population. Introducing (cf. (2.2)) the individual growth rate

$$
g\left(x, Z_{1}\right)=\eta_{1}\left(\frac{E_{1}(x) C_{1}(x) Z_{1}}{1+H_{1}(x) C_{1}(x) Z_{1}}-\zeta_{1} x\right),
$$

we can express the probability $\Pi\left(Z_{1}\right)$, that a newborn individual survives till it reaches size $x_{A}$ and becomes adult, given a constant (in time) food concentration $Z_{1}$, as

$$
\Pi\left(Z_{1}\right)=\exp \left(-\int_{x_{b}}^{x_{A}} \frac{\mu_{1}(y)}{g\left(y, Z_{1}\right)} d y\right) .
$$

Once turned adult, the individual lives for an expected period $\frac{1}{\mu_{2}}$ of time, during which it produces offspring at the rate (2.4) when adult food has the constant (in time) concentration $Z_{2}$. So the expected number of offspring produced by an individual just turned adult equals

$$
R_{A}\left(Z_{2}\right)=\frac{1}{\mu_{2}} \eta_{2}\left(\frac{E_{2} C_{2} Z_{2}}{1+H_{2} C_{2} Z_{2}}-\zeta_{2} x_{A}\right) .
$$

Consequently the expected number of offspring produced by a newborn individual equals

$$
R_{0}\left(Z_{1}, Z_{2}\right)=\Pi\left(Z_{1}\right) R_{A}\left(Z_{2}\right) .
$$


A first requirement for steady state is that the two food concentrations are such that

$$
R_{0}\left(Z_{1}, Z_{2}\right)=1 \text {. }
$$

If, under such conditions, the steady population birth rate equals $c$, the stable juvenile size distribution is described by the density function

$$
n(x)=\frac{c}{g\left(x, Z_{1}\right)} \exp \left(-\int_{x_{b}}^{x} \frac{\mu_{1}(y)}{g\left(y, Z_{1}\right)} d y\right) .
$$

Therefore, the rate $g\left(x_{A}, Z_{1}\right) n\left(x_{A}\right)$ at which adults are recruited, equals $c \Pi\left(Z_{1}\right)$ and we have

$$
N_{A}=\frac{c \Pi\left(Z_{1}\right)}{\mu_{2}} .
$$

The second and third requirements for steady state are, that the left hand sides of (2.5) and (2.6) are equal to zero, therefore, using (3.6) and (3.7), we find

$$
\begin{aligned}
& 0=r_{1}\left(K_{1}-Z_{1}\right)-c Z_{1} \int_{x_{b}}^{x_{A}} \frac{C_{1}(x)}{1+H_{1}(x) C_{1}(x) Z_{1}} \frac{1}{g\left(x, Z_{1}\right)} e^{-\int_{x_{b}}^{x} \frac{\mu_{1}(y)}{g\left(y, Z_{1}\right)} d y} d x, \\
& 0=r_{2}\left(K_{2}-Z_{2}\right)-\frac{C_{2} Z_{2}}{1+H_{2} C_{2} Z_{2}} \frac{c \Pi\left(Z_{1}\right)}{\mu_{2}} .
\end{aligned}
$$

Together (3.5), (3.8) and (3.9) are three equations in the three unknowns $Z_{1}, Z_{2}$ and $c$. For any solution (with $c, Z_{i} \geq 0$ ) the steady population size and composition is given by (3.6), (3.7), while the $Z_{i}$ are the steady food levels. By eliminating $c$ from (3.8), (3.9) one reduces the system to two equations in the two unknowns $Z_{1}$ and $Z_{2}$. Each equation defines a curve in the $\left(Z_{1}, Z_{2}\right)$-plane and solutions are precisely the points of intersection of the two curves.

\section{The steady state equations for a cannibalistic population}

For a cannibalistic population the situation becomes more complicated. Yet, following the methodology introduced in (Diekmann et al. 2003), we can derive six equations in six unknowns. The key idea is to rewrite (2.7) and (2.8) as, respectively,

$$
\frac{E_{2} C_{2} Z_{2}+I_{1}}{1+H_{2} C_{2} Z_{2}+I_{2}}
$$

and

$$
\mu_{1}(x)+\frac{C_{x} I_{3}}{1+H_{2} C_{2} Z_{2}+I_{2}}
$$

introducing

$$
\begin{aligned}
I_{1} & :=\int_{x_{b}}^{x_{A}} E_{y} C_{y} n(y) d y, \\
I_{2} & :=\int_{x_{b}}^{x_{A}} H_{y} C_{y} n(y) d y, \\
I_{3} & :=N_{A} .
\end{aligned}
$$

The two following points are crucial: 
1) The dependence on the infinite-dimensional parameter $n=n(y)$ in (2.7) and (2.8) is replaced by a dependence on the three dimensional variable $\left(I_{1}, I_{2}, I_{3}\right)$ in (4.1) and (4.2). So the $I_{i}$ capture exactly the feedback of the population on the individuals. We call them interaction variables and we call the vector $I$ environmental condition (where one should realize that we do not explicitly consider aspects of the "true" environment that are not in one way or another under the influence of feedback).

2) The $I_{i}$ are chosen such that their dependence on $n$ in (4.3) and (4.4) is linear.

A consequence of point 1) is that when constructing the expected lifetime offspring $R_{0}$ from the energy uptake (4.1) and mortality (4.2) we obtain a condition for steady state of the form

$$
R_{0}(I)=1
$$

(see (4.11) below), i.e., an equation in a finite number of variables. To see the advantage resulting from 2), note first that the expression (3.6) for the juvenile size density is replaced by

$$
n(x)=\frac{c}{g\left(x, Z_{1}\right)} \exp \left(-\int_{x_{b}}^{x}\left(\mu_{1}(y)+\frac{C_{y} I_{3}}{1+H_{2} C_{2} Z_{2}+I_{2}}\right) \frac{1}{g\left(y, Z_{1}\right)} d y\right)
$$

and (3.7) now reads

$$
N_{A}=I_{3}=\frac{c \Pi^{c}\left(Z_{1}, Z_{2}, I_{2}, I_{3}\right)}{\mu_{2}},
$$

where $\Pi^{c}$ is defined in (4.9) below and the superindex $c$ refers to cannibalism (and not to the population birth rate). Note in particular that both $n$ and $I_{3}$ depend linearly on $c$. If we plug (4.6) into (4.3) and (4.4) the same applies to $I_{1}$ and $I_{2}$. Thus we obtain equations of the form

$$
I_{i}=c F\left(Z_{1}, Z_{2}, I_{1}, I_{2}, I_{3}\right), \quad i=1,2,3 .
$$

We will supplement the four equations (4.5) and (4.8) by two more equations expressing that the food concentrations $Z_{1}$ and $Z_{2}$ should be steady. Thus we obtain six equations in six unknowns, $c, I_{1}, I_{2}, I_{3}$ and $Z_{1}$ and $Z_{2}$. In summary, we have arrived at a finite dimensional problem, where the linear $c$-dependence in (4.8) is tailor-made for the bifurcation analysis that we will perform in Section 6.

The steady state is completely determined by a steady environmental condition $I$ and a population birth rate $c$, see (4.6) and (4.7). The equations that these quantities should satisfy are consistency conditions. Solving the steady state problem can thus be interpreted as specifying an environment $I$ and a "population" $c$ such that the basic reproduction number under this environmental condition equals one and such that the population consistently determines the environment. 
One of the remaining tasks is hence to model $R_{0}$ and in order to do so observe, that the analogue of (3.2) is

$$
\Pi^{c}\left(Z_{1}, Z_{2}, I_{2}, I_{3}\right)=\exp \left(-\int_{x_{b}}^{x_{A}}\left(\mu_{1}(x)+\frac{C_{x} I_{3}}{1+H_{2} C_{2} Z_{2}+I_{2}}\right) \frac{1}{g\left(x, Z_{1}\right)} d x\right)
$$

and the analogue of (3.3) is

$$
R_{A}^{c}\left(Z_{2}, I_{1}, I_{2}\right)=\frac{1}{\mu_{2}} \eta_{2}\left(\frac{E_{2} C_{2} Z_{2}+I_{1}}{1+H_{2} C_{2} Z_{2}+I_{2}}-\zeta_{2} x_{A}\right)
$$

Thus, (3.5) becomes

$$
R_{0}^{c}\left(Z_{1}, Z_{2}, I_{1}, I_{2}, I_{3}\right)=1
$$

where by definition

$$
R_{0}^{c}\left(Z_{1}, Z_{2}, I_{1}, I_{2}, I_{3}\right)=\Pi^{c}\left(Z_{1}, Z_{2}, I_{2}, I_{3}\right) R_{A}^{c}\left(Z_{2}, I_{1}, I_{2}\right) .
$$

The conditions for steady food levels are

$$
\begin{aligned}
0= & r_{1}\left(K_{1}-Z_{1}\right)-c Z_{1} \int_{x_{b}}^{x_{A}} \frac{C_{1}(x)}{1+H_{1}(x) C_{1}(x) Z_{1}} \frac{1}{g\left(x, Z_{1}\right)} \\
& \times \exp \left(-\int_{x_{b}}^{x}\left(\mu_{1}(y)+\frac{C_{y} I_{3}}{1+H_{2} C_{2} Z_{2}+I_{2}}\right) \frac{1}{g\left(y, Z_{1}\right)} d y\right) d x, \\
0= & r_{2}\left(K_{2}-Z_{2}\right)-\frac{C_{2} Z_{2} I_{3}}{1+H_{2} C_{2} Z_{2}+I_{2}} .
\end{aligned}
$$

The equations (4.11), (4.13) and (4.14) are supplemented by the so called feedback relations (4.7) and

$$
\begin{aligned}
I_{1}= & c \int_{x_{b}}^{x_{A}} \frac{E_{x} C_{x}}{g\left(x, Z_{1}\right)} \\
& \times \exp \left(-\int_{x_{b}}^{x}\left(\mu_{1}(y)+\frac{C_{y} I_{3}}{1+H_{2} C_{2} Z_{2}+I_{2}}\right) \frac{1}{g\left(y, Z_{1}\right)} d y\right) d x, \\
I_{2}= & c \int_{x_{b}}^{x_{A}} \frac{H_{x} C_{x}}{g\left(x, Z_{1}\right)} \\
& \times \exp \left(-\int_{x_{b}}^{x}\left(\mu_{1}(y)+\frac{C_{y} I_{3}}{1+H_{2} C_{2} Z_{2}+I_{2}}\right) \frac{1}{g\left(y, Z_{1}\right)} d y\right) d x .
\end{aligned}
$$

Together, (4.11) and (4.7)-(4.16) constitute six equations in the six unknowns $c$, $Z_{1}, Z_{2}, I_{1}, I_{2}$ and $I_{3}$. 


\section{The evolutionary onset of cannibalism}

Suppose, that the food levels are set at constant values by a non-cannibalistic population. Suppose a cannibalistic mutant enters the scene and suppose that it reproduces clonally. Does it generate an exponentially growing clan or does its clan go extinct? Ignoring the subtleties of demographic stochasticity, the answer is provided by the quantity $R_{0}^{c}\left(Z_{1}, Z_{2}, I_{1}, I_{2}, 0\right)$ defined by (4.12), in the sense that exponential growth sets in if and only if it exceeds one. The fact that we have put $I_{3}$ equal to zero correponds to the assumption, that as long as the clan is small relative to the population at large, the chance that its juvenile members fall victim to cannibalism is negligible. Therefore, the only possible disadvantage a cannibalistic invader faces, is that while cannibalising it loses time it could spend on a possibly more nutritious recource. Likewise one should put $I_{3}=0$ in the defining expressions (4.15), (4.16) for $I_{1}$ and $I_{2}$. As a result we have explicit formulas for $I_{1}$ and $I_{2}$ in terms of $c, Z_{1}$ and $Z_{2}$. Consequently, $R_{0}^{c}\left(Z_{1}, Z_{2}, I_{1}, I_{2}, 0\right)$ is a computable number, once the $c$, $Z_{1}$ and $Z_{2}$ are specified.

Now note, that from (4.9), it follows that $\Pi^{c}\left(Z_{1}, Z_{2}, I_{2}, 0\right)$ is independent of $I_{2}$ and $Z_{2}$ and, in fact, equal to $\Pi\left(Z_{1}\right)$ defined in (3.2). Hence $R_{0}^{c}\left(Z_{1}, Z_{2}, I_{1}, I_{2}, 0\right)>1$ if and only if

$$
R_{A}^{c}\left(Z_{2}, I_{1}, I_{2}\right)>R_{A}\left(Z_{2}\right)
$$

(since $\left.R_{0}^{c}\left(Z_{1}, Z_{2}, 0,0,0\right)=\Pi\left(Z_{1}\right) R_{A}\left(Z_{2}\right)=1\right)$. Therefore, the cannibalistic subpopulation will start to grow exponentially in the environmental conditions as set by the non-cannibalistic resident if and only if

$$
\frac{E_{2} C_{2} Z_{2}+I_{1}}{1+H_{2} C_{2} Z_{2}+I_{2}}>\frac{E_{2} C_{2} Z_{2}}{1+H_{2} C_{2} Z_{2}} .
$$

This simply means, that the cannibalistic energy intake and handling time should compensate each other in such a way, that the "overall" energy intake rate of a cannibal exceeds the one of a non-cannibal. Now, inequality (5.2) can also be written as

$$
\frac{I_{1}}{I_{2}}>\frac{E_{2} C_{2} Z_{2}}{1+H_{2} C_{2} Z_{2}},
$$

which means that the ratio of cannibalism related energy intake and cannibalism related handling time should exceed the average energy intake rate of non-cannibalistic individuals. Hence, we deduced the standard optimal foraging criterion. A necessary condition for (5.3) to hold is, that

$$
\frac{E_{y}}{H_{y}}>\frac{E_{2} C_{2} Z_{2}}{1+H_{2} C_{2} Z_{2}},
$$

for at least some $y \in\left(x_{b}, x_{A}\right)$. This is simply a size-specific version of the criterion. If it is satisfied, one can satisfy (5.3) by restricting $C_{y}$ such that it is positive only for $y$ for which (5.4) holds (whether or not this is a physiologically feasible option is another matter). 
We conclude that the evolutionary onset of cannibalism can be understood via optimal foraging considerations. (And, actually, so is a further intensification of cannibalistic activity: a rare type of individual does not suffer from the extra mortality it generates by direct interaction, since this is a quadratic effect).

\section{Population level effects}

The lifeboat mechanism refers to the possibility that the steady state equations, i.e., (4.11), (4.7)-(4.16), may have a solution for values of the carrying capacity parameters $K_{1}$ and $K_{2}$ for which (3.5), (3.8), (3.9) does not have a solution, i.e., values where, as opposed to a cannibalistic population, a noncannibalistic population can not persist. To investigate this possibility, we focus on a neighbourhood of the critical set in the $\left(K_{1}, K_{2}\right)$-plane, where $R_{0}=1$ for a population which invades the virgin environment in which both food levels are at their carrying capacities. Since, as indeed should be the case,

$$
R_{0}^{c}\left(Z_{1}, Z_{2}, 0,0,0\right)=R_{0}\left(Z_{1}, Z_{2}\right)
$$

the critical set is the same for the non-cannibalistic as for the cannibalistic population. It is defined by the equation

$$
R_{0}\left(K_{1}, K_{2}\right)=1
$$

Since (cf. (3.4)) $R_{0}\left(K_{1}, K_{2}\right)$ is the product of two monotone functions of a single variable, the critical set has the qualitative features depicted in Figure 1. For the noncannibalistic population, a steady state can only exist, if $\left(K_{1}, K_{2}\right)$ lies above the critical curve, because from (3.8) and (3.9) we see, that $c>0$ implies $Z_{i}<K_{i}$ for $i \in\{1,2\}$ whence $\Pi\left(K_{1}\right)>\Pi\left(Z_{1}\right)$ and $R_{A}\left(K_{2}\right)>R_{A}\left(Z_{2}\right)$ and, consequently,

$$
R_{0}\left(K_{1}, K_{2}\right)>R_{0}\left(Z_{1}, Z_{2}\right)=1 .
$$

For the cannibalistic population it is not as simple to determine the set of $\left(K_{1}, K_{2}\right)$ values for which a non-trivial steady state (i.e., $\left.c>0\right)$ exists. Our strategy is to concentrate on small values of $c$ and to do a perturbation analysis. More precisely, in the six equations (4.11), (4.7)-(4.16), we shall consider $I=\left(I_{1}, I_{2}, I_{3}\right)$, $Z=\left(Z_{1}, Z_{2}\right)$ and $K_{2}$ as the unknowns and $K_{1}$ and $c$ as parameters. For $c=0$ we have the one parameter curve of solutions $I=0, Z=K, K_{2}=\Phi_{0}\left(K_{1}\right)$, where $\Phi_{0}$ is the function whose graph is depicted in Figure 1 or, in other words

$$
\Phi_{0}\left(K_{1}\right):=R_{A}^{-1}\left(\frac{1}{\Pi\left(K_{1}\right)}\right) .
$$

Now, fix $K_{1}$ and look for solutions of the form

$$
\begin{aligned}
I & =c k\left(K_{1}\right)+o(c), \\
Z & =K+c \Theta\left(K_{1}\right)+o(c), \\
K_{2} & =\Phi_{0}\left(K_{1}\right)+c \Phi_{1}\left(K_{1}\right)+o(c),
\end{aligned}
$$




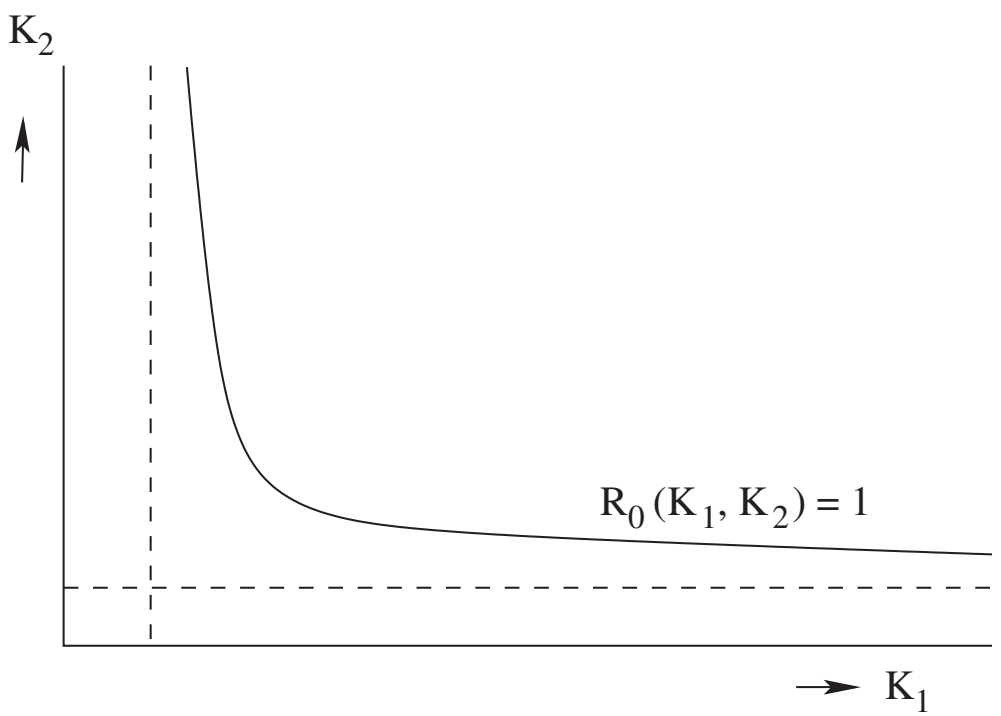

Fig. 1. Graph of the critical curve $R_{0}\left(K_{1}, K_{2}\right)=1$. For parameters in the region above the curve, a population introduced in the virgin environment $Z=K$ starts growing exponentially, while for parameters in the region below the curve, a small population goes extinct. The asymptotic values are defined as the solutions of $\lim _{K_{1} \uparrow \infty} \Pi\left(K_{1}\right) R_{A}\left(K_{2}\right)=$ $1, \lim _{K_{2} \uparrow \infty} \Pi\left(K_{1}\right) R_{A}\left(K_{2}\right)=1$.

where $o(c)$ denote the higher order terms in the small parameter $c$. As emphasized in (Diekmann et al. 2003), our way of pre-processing the steady state problem has the advantage, that $k$ and $\Theta$ are easily computed. Indeed, from, respectively, (4.15), (4.16) and (4.7), we deduce

$$
k\left(K_{1}\right)=\left(\begin{array}{c}
\int_{x_{b}}^{x_{A}} \frac{E_{x} C_{x}}{g\left(x, Z_{1}\right)} e^{-\int_{x_{b}}^{x} \frac{\mu_{1}(y)}{g\left(y, Z_{1}\right)} d y} d x \\
\int_{x_{b}}^{x_{A}} \frac{H_{x} C_{x}}{g\left(x, Z_{1}\right)} e^{-\int_{x_{b}}^{x} \frac{\mu_{1}(y)}{g\left(y, Z_{1}\right)} d y} d x \\
\frac{\Pi\left(K_{1}\right)}{\mu_{2}}
\end{array}\right) .
$$

Likewise, we deduce from (4.13) and (4.7), plugged into (4.14), that

$$
\Theta\left(K_{1}\right)=-\left(\begin{array}{c}
\frac{K_{1}}{r_{1}} \int_{x_{b}}^{x_{A}} \frac{C_{1}(x)}{1+H_{1}(x) C_{1}(x) K_{1}} \frac{1}{g\left(x, K_{1}\right)} e^{-\int_{x_{b}}^{x} \frac{\mu_{1}(y)}{g\left(y, K_{1}\right)} d y} d x \\
\frac{1}{r_{2}} \frac{C_{2} \Phi_{0}\left(K_{1}\right)}{1+H_{2} C_{2} \Phi_{0}\left(K_{1}\right)} \frac{\Pi\left(K_{1}\right)}{\mu_{2}}
\end{array}\right) .
$$

It remains to determine $\Phi_{1}\left(K_{1}\right)$. To do so, in (6.4) we plug the third equation into the second, then the first two into (4.11) and finally expand up to first order with respect to $c$. This way, we obtain the equation

$$
\frac{\partial R_{0}^{c}}{\partial Z_{2}} \Phi_{1}\left(K_{1}\right)+\frac{\partial R_{0}^{c}}{\partial Z} \Theta\left(K_{1}\right)+\frac{\partial R_{0}^{c}}{\partial I} k\left(K_{1}\right)=0
$$


which is linear in $\Phi_{1}, \Theta$ and $k$ (note that the last two terms are vector products). It can be solved for $\Phi_{1}$, since monotonicity guarantees, that $\frac{\partial R_{0}^{c}}{\partial Z_{2}} \neq 0$ (note that, for the same reason, our formal solution is justified by the Implicit Function Theorem).

Since $\frac{\partial R_{0}^{c}}{\partial Z_{2}}>0$, we deduce that

$$
\operatorname{sign} \Phi_{1}=-\operatorname{sign}\left(\frac{\partial R_{0}^{c}}{\partial Z} \Theta+\frac{\partial R_{0}^{c}}{\partial I} k\right) .
$$

Since $K_{2} \longrightarrow R_{0}\left(K_{1}, K_{2}\right)$ is monotonically increasing, it follows from the last equation of (6.4), that the small-c-level steady state solution exists in the region where

$$
R_{0}\left(K_{1}, K_{2}\right)<1
$$

if and only if sign $\Phi_{1}<0$. Therefore we will say from now on, that the life boat mechanism works when sign $\Phi_{1}<0$. Note, that by speaking about small-c-level solutions, we emphasize that this is a local property: we refer to (Van den Bosch et al. 1988) for an example of more complicated global behaviour.

\section{Elaboration of the criteria}

In this section we consider (5.3) and (6.8) in more detail, both separately and to uncover their interrelationship.

At the critical curve $K_{2}=\Phi_{0}\left(K_{1}\right)$, the left hand side of (5.3) reduces to

$$
\frac{\int_{x_{b}}^{x_{A}} \frac{E_{y} C_{y}}{g\left(y, K_{1}\right)} e^{-\int_{x_{b}}^{y} \frac{\mu_{1}(x)}{g\left(x, K_{1}\right)} d x} d y}{\int_{x_{b}}^{x_{A}} \frac{H_{y} C_{y}}{g\left(y, K_{1}\right)} e^{-\int_{x_{b}}^{y} \frac{\mu_{1}(x)}{g\left(x, K_{1}\right)} d x} d y}
$$

and therefore the no-onset condition, i.e., the reverse of (5.3), can be formulated as

$$
\int_{x_{b}}^{x_{A}}\left(\frac{E_{y}}{H_{y}}-\frac{E_{2} C_{2} \Phi_{0}\left(K_{1}\right)}{1+H_{2} C_{2} \Phi_{0}\left(K_{1}\right)}\right) \frac{H_{y} C_{y}}{g\left(y, K_{1}\right)} e^{-\int_{x_{b}}^{y} \frac{\mu_{1}(x)}{g\left(x, K_{1}\right)} d x} d y<0 .
$$

Accordingly, a sufficient no-onset condition is, that

$$
\frac{E_{y}}{H_{y}}<\frac{E_{2} C_{2} \Phi_{0}\left(K_{1}\right)}{1+H_{2} C_{2} \Phi_{0}\left(K_{1}\right)}
$$

for all $y$ which correspond to vulnerable juveniles (or, in mathematical jargon, for all $y$ in the support of $C_{y}$ ).

As $\Phi_{0}$ is a decreasing function, we conclude that,

if the sufficient no-onset condition (7.2) is satisfied for some $K_{1}$, it is satisfied for all smaller values of $K_{1}$ (nota bene that, along the curve, $K_{1}$ and $K_{2}$ are coupled!).

If we additionally assume, that $\frac{E_{y}}{H_{y}}$ is the same for all vulnerable juveniles, we can say a little more. First, note that $\Phi_{0}\left(K_{1}\right)$ tends, for $K_{1} \longrightarrow \infty$, to the value of 
$Z_{2}$, which makes $R_{A}$ equal to $\frac{1}{\Pi(\infty)}$ (recall Figure 1$)$. Therefore, we deduce from (3.3), that

$$
\frac{E_{2} C_{2} \Phi_{0}\left(K_{1}\right)}{1+H_{2} C_{2} \Phi_{0}\left(K_{1}\right)}=\zeta_{2} x_{A}+\frac{\mu_{2}}{\eta_{2} \Pi(\infty)} .
$$

Thus, a necessary condition for onset of cannibalism is

$$
\frac{E_{y}}{H_{y}}>\zeta_{2} x_{A}+\frac{\mu_{2}}{\eta_{2} \Pi(\infty)}
$$

for vulnerable juveniles.

At the other extreme, where $K_{1}$ tends to the lower boundary of the interval on which $\Phi_{0}$ is defined, we have $\Phi_{0}\left(K_{1}\right) \longrightarrow \infty$. Therefore, if

$$
\frac{E_{y}}{H_{y}}>\frac{E_{2}}{H_{2}},
$$

the necessary onset condition is satisfied for all values of $K_{1}$, for which the population can persist. Note, that implicitly we have assumed, that

$$
\frac{E_{2}}{H_{2}}>\zeta_{2} x_{A}+\frac{\mu_{2}}{\eta_{2} \Pi(\infty)},
$$

since otherwise this set of $K_{1}$ values would be empty. Hence, (7.5) is indeed a more severe condition than (7.4). The meaning of (7.5) is, that cannibalistic food is more profitable than standard adult food. If (7.5) is not satisfied, but (7.4) is, we show that there is along the critical curve exactly one point, such that onset is impossible to the left, but possible to the right (see Figure 2).

Above the critical curve, the inequality (5.2) can be rewritten as

$$
\int_{x_{b}}^{x_{A}}\left(\frac{E_{y}}{H_{y}}-\frac{E_{2} C_{2} Z_{2}}{1+H_{2} C_{2} Z_{2}}\right) \frac{H_{y} C_{y}}{g\left(y, Z_{1}\right)} e^{-\int_{x_{b}}^{y} \frac{\mu_{1}(x)}{g\left(x, Z_{1}\right)} d x} d y>0 .
$$

Still assuming that $\frac{E_{y}}{H_{y}}$ is constant and that (7.4), but not (7.5), is satisfied, we will obtain a well-defined critical $Z_{2}$ level, at which the left hand side of (7.6) switches sign. More precisely, let $Z_{2}^{c}$ denote the unique solution of the equation

$$
\frac{E_{y}}{H_{y}}=\frac{E_{2} C_{2} Z_{2}}{1+H_{2} C_{2} Z_{2}},
$$

then onset is impossible, if $Z_{2}^{s s}>Z_{2}^{c}$ and possible if $Z_{2}^{s s}<Z_{2}^{c}$, where $Z_{2}^{s s}$ is the steady state adult food level. Explicitly, from (7.7) we get

$$
Z_{2}^{c}=\frac{E_{y}}{C_{2}\left(E_{2} H_{y}-H_{2} E_{y}\right)} .
$$

Our next aim is to find the curve in the $\left(K_{1}, K_{2}\right)$-parameter plane, at which $Z_{2}^{s s}=$ $Z_{2}^{c}$. We shall find, that this is a straight line with positive slope, starting at the point on the critical curve described above and defined (uniquely) by $\Phi_{0}\left(K_{1}\right)=Z_{2}^{c}$. 


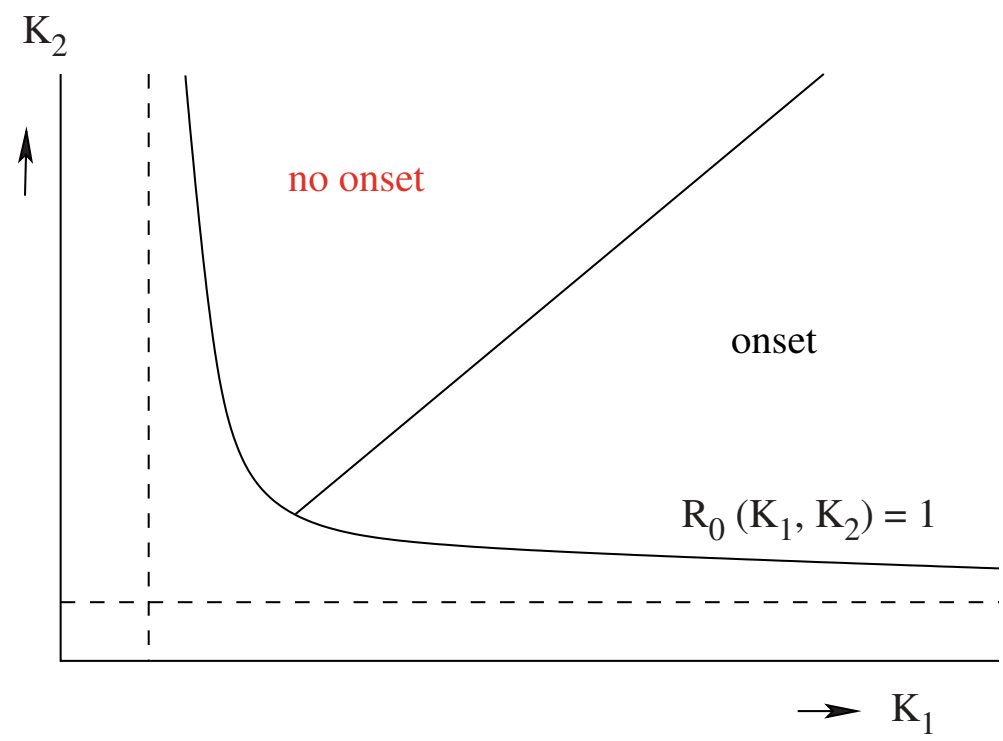

Fig. 2. Subdivision of the parameter plane in the region in which onset of cannibalism is impossible and the complementary region in which onset of cannibalism is possible.

If the level of $Z_{2}$ is fixed a priori, we can use (3.5) to determine the level of $Z_{1}$. Thus, define

$$
Z_{1}^{c}=\Pi^{-1}\left(\frac{1}{R_{A}\left(Z_{2}^{c}\right)}\right) .
$$

Next, use (3.9) to determine $c$. The result is

$$
c=r_{2} \frac{E_{2}}{E_{y}} R_{A}\left(Z_{2}^{c}\right) \mu_{2} H_{y}\left(K_{2}-Z_{2}^{c}\right) .
$$

Finally, substituting (7.9) and (7.10) into (3.8), we find a linear equation relating $K_{1}$ and $K_{2}$. In the equation, the coefficient of $K_{1}$ is positive and the coefficient of $K_{2}$ is negative, therefore the graph of $K_{2}$ as a function of $K_{1}$ is a straight line with positive slope. Monotonicity arguments guarantee that onset is possible to the right of this line and impossible to the left (see Figure 2).

We now turn our attention to (6.8) and first make the following sign observations:

$$
\frac{\partial R_{0}^{c}}{\partial Z_{1}}>0, \quad \frac{\partial R_{0}^{c}}{\partial Z_{2}}>0, \quad \frac{\partial R_{0}^{c}}{\partial I_{1}}>0, \quad \frac{\partial R_{0}^{c}}{\partial I_{2}}<0, \quad \frac{\partial R_{0}^{c}}{\partial I_{3}}<0,
$$

where all quantities are evaluated at the critical curve, such that $Z_{1}=K_{1}, Z_{2}=$ $\Phi_{0}\left(K_{1}\right)$ and $I=0$. In addition, from (6.5) and (6.6) one sees that

$$
\theta_{i}<0, \quad k_{i}>0,
$$


so only

$$
\frac{\partial R_{0}^{c}}{\partial I_{1}} k_{1}
$$

(corresponding to extra energy intake of adults) gives a positive contribution to $\frac{\partial R_{0}^{c}}{\partial Z} \theta+\frac{\partial R_{0}^{c}}{\partial I} k$, all other terms are negative. The term $\frac{\partial R_{0}^{c}}{\partial I_{2}} k_{2}$ corresponds to the diminishing of the energy intake of standard food due to time/digestion capacity spent on intraspecific predation, $\frac{\partial R_{0}^{c}}{\partial I_{3}} k_{3}$ to the decreased survival probability due to cannibalistic predation pressure and the $\frac{\partial R_{0}^{c}}{\partial Z_{i}} \Theta_{i}$ to the increase of energy intake with standard food. Now note that from (4.12), (4.9) and (4.10) one computes

$$
\begin{aligned}
& \frac{\partial R_{0}^{c}}{\partial I_{1}} k_{1}+\frac{\partial R_{0}^{c}}{\partial I_{2}} k_{2} \\
& =\frac{\eta_{2} \Pi\left(K_{1}\right)}{\mu_{2}\left(1+H_{2} C_{2} \Phi_{0}\left(K_{1}\right)\right)} \\
& \quad \int_{x_{b}}^{x_{A}}\left(\frac{E_{y}}{H_{y}}-\frac{E_{2} C_{2} \Phi_{0}\left(K_{1}\right)}{1+H_{2} C_{2} \Phi_{0}\left(K_{1}\right)}\right) \frac{H_{y} C_{y}}{g\left(y, K_{1}\right)} e^{-\int_{x_{b}}^{y} \frac{\mu_{1}(x)}{g\left(x, K_{1}\right)} d x} d y
\end{aligned}
$$

Comparison with (7.1) and/or (7.2) immediately leads to the conclusion, that the life boat mechanism can only work when onset of cannibalism is possible. Since the quantity in (7.11) needs to be sufficiently positive to compensate the negative contributions of the other three terms in

$$
\frac{\partial R_{0}^{c}}{\partial Z} \theta+\frac{\partial R_{0}^{c}}{\partial I} k
$$

(in order for $\Phi_{1}$ to be negative; recall (6.8)), we see clearly, that a more severe condition needs to be satisfied for the life boat mechanism to work, than for mere onset of cannibalism. Note, that unlike in the limit $K_{2} \longrightarrow \infty$, for $K_{1} \longrightarrow \infty$ the sign of $\Phi$ is not apparent, without further assumptions. We suspect, that the quantity $\Phi_{1}$ switches sign at most once along the critical curve, but we did not manage to prove this. A sufficient condition for the lifeboat mechanism, however, can be given, by observing, that $\frac{E_{y}}{H_{y}}$ (assumed constant) appears only once in (7.11) and not at all in the other terms of

$$
\frac{\partial R_{0}^{c}}{\partial Z} \theta+\frac{\partial R_{0}^{c}}{\partial I} k .
$$

It follows that the lifeboat mechanism manifests itself for sufficiently large $\frac{E_{y}}{H_{y}}$. We refer to (Diekmann et al. 2003) and (Getto, thesis) for a further elaboration of the life boat condition in the simpler setting of constant food availability and for a precise biological interpretation of the condition in such a setting. 


\section{Concluding remarks}

In the context of a size structured population model with two dynamic food sources, one for juveniles and one for adults, we have investigated both the conditions for the evolutionary onset of cannibalism and the condition for persistence of a cannibalistic population, where it would go extinct in the absence of cannibalism. At a qualitative level the conditions agree, in quantitative detail they differ considerably. The common denominator is, that cannibalism, in the form that the big adults eat the small juveniles, pays when adult food is limiting and does not pay when juvenile food is limiting.

Our adaptive dynamics approach focussed on steady state attractors, mainly since these are amenable to analysis with pencil and paper. We do think, however, that the distinction between onset of cannibalism and the life boat mechanism might become negligible when food fluctuates. To verify this idea one could, for instance, repeat the analysis of this paper for the situation that one or both of the parameters $K_{1}$ and $K_{2}$ is a periodic function of time. Another possibility is, to assume that reproduction is concentrated in a short period of the year. Even with constant $K$ the food levels will then change during the year, due to the physiological development of the cohorts.

In a recent review on the population dynamic theory of size-dependent cannibalism, (Claessen et al. 2004) distinguished four critical aspects of cannibalism: 1) victim mortality, 2) energy extraction from victims, 3) size dependence and 4) competition. The size dependence of the cannibalistic interaction results from that cannibals are usually larger than their victims, while competition is implicated because cannibals and victims are the same species and may hence share common resources. Of the 30 different models of cannibalistic interactions reviewed, all accounted for victim mortality, but only $50 \%$ of them accounted for the direct positive effect of energy gain from killing and eating a conspecific. Few models accounted for all four aspects of cannibalism. At the population level different types of possible responses due to cannibalism were distinguished, among which the occurrence of multiple equilibria and the life boat effect. (Cushing 1991) pointed out already that the interplay between positive and negative effects of cannibalism can result in multiple steady states. Energy extraction from victims is the direct, positive effect, but indirect, positive effects occur as well, as cannibalism may reduce intraspecific competition and hence benefit growth and reproduction. (Claessen et al. 2004) argue that the life boat effect results from the disbalance between the direct, positive effect of energy extraction and the direct, negative effect of imposing victim mortality. The model we analyze does account for victim mortality, energy extraction and size-dependence of the cannibalistic interaction. The fourth aspect of cannibalism, resource competition between cannibals and victims, does not play as much of a role as we focus on situations of low population density and moreover assume that juveniles and adults have exclusive resources. However, in contrast to (Claessen et al. 2004) we recognise that there are additional, indirect negative effects influencing the occurrence of the life boat mechanism: any increase in density translates into a decrease in resources and hence to a slowing down of juvenile growth. Moreover, by predating conspecifics a cannibal can forage less on alternative resources, 
as it has less time available to search for it. To our knowledge these indirect, negative effects, which are embodied in the condition (6.8), have not been recognised before. The condition for the occurrence of the life boat effect is hence necessarily more complicated than proposed by (Van den Bosch et al. 1988), who argued that it occurred whenever the expected energy gain from cannibalizing a single juvenile is sufficient to produce a new single offspring (see Section 4.1 of (Diekmann et al. 2003) for explicit incorporation of the subtle aspect that those juveniles that are cannibalized, but wouldn't have reached adulthood anyhow because of death of other causes, come for free). (Polis 1981) proposed that the persistence of cannibalistic populations under conditions under which non-cannibalistic populations go extinct results from that adult individuals by cannibalizing offspring indirectly gain access to a juvenile resource base, which is otherwise inaccessible to them. The condition (6.8) relates to that, since it clearly expresses that the life boat effect will only occur whenever adult resources are scarce and the adults require the indirect access to juvenile resources for persistence. Our analysis shows furthermore that cannibalism may evolve under a wider range of conditions, independent of the population level effect. However, both the onset of cannibalism and the life boat effect do depend crucially on the direct positive effect of cannibalism, the energy gain from eating a smaller conspecific, which should be contrasted with the fact that half of the previous models on cannibalism do not take this positive aspect into account, including the well-studied model of the population dynamics of Tribolium (Costantino et al. 1997).

Acknowledgements. During the final stages, the research of Philipp Getto was supported by the project MRTN-CT-2004-503661: Modeling, Mathematical Methods and Computer Simulation of Tumour Growth and Therapy.

\section{References}

Botsford, L.W.: The effects of increased individual growth rates on depressed population size. Am. Nat. 117, 38-63 (1981)

Claessen, D., Dwarfs, Giants: The dynamic interplay of size-dependent cannibalism and competition. Ph. D. thesis.

Claessen, D., de Roos, A.M.: Bistability in a size-structured population model of cannibalistic fish- a continuation study. Theor. Pop. Biol. 64 (1), 49-65 (2003)

Claessen, D., de Roos, A.M., Persson, L.: Population dynamic theory of size-dependent cannibalism. Proc. R. Soc. Lond. B. 271, 333-340 (2004)

Costantino, R.F., Desharnais, R.A., Cushing, J.M., Dennis, B.: Chaotic dynamics in an insect population. Science. 275, 389-391 (1997)

Cushing, J.M.: A simple model of cannibalism. Math. Biosci. 107, 47-71 (1991)

Dieckmann, U., Law, R.: The dynamical theory of coevolution: a derivation from stochastic ecological processes. J. Math. Biol. 34, 579-612 (1996)

Diekmann, O.: Modeling and analysing physiologically structured populations. In: Capasso, V., Diekmann, O., (eds.) Mathematics inspired by Biology, Springer, LN. M 1714 (1999)

Diekmann, O.: A beginners guide to adaptive dynamics. Banach Center Publications 63, 47-86 (2004)

Diekmann, O., Gyllenberg, M., Metz, J.A.J.: Steady State Analysis of Structured Population Models. Theor. Popul. Biol. 63, 309-338 (2003)

Geritz, S.A.H., Metz, J.A.J., Kisdi, É., Meszéna, G.: The dynamics of adaptation and evolutionary branching. Phys. Rev. Letters 78, 2024-2027 (1997) 
Geritz, S.A.H., Kisdi, É., Meszéna, G., Metz, J.A.J.: Evolutionarily singular strategies and the adaptive growth and branching of the evolutionary tree. Evol. Ecol. 12, 35-57 (1998)

Getto, Ph.: Steady state analysis for a size structured cannibalism model with two dynamic resources. In: On some quasilinear structured population models, Ph. D. thesis. http://igiturarchive.library.uu.nl/dissertations/2005-0816-200002/index.htm

Henson, S.M.: Cannibalism can be beneficial even when its mean yield is less than one. Theor. Popul. Biol. 51, 107-117 (1997)

Metz, J.A.J., Nisbet, R.M., Geritz, S.A.H.: How should we define "fitness" for general ecological scenarios? TREE 7, 198-202 (1992)

Metz, J.A.J., Geritz, S.A.H., Meszéna, G., Jacobs, F.J.A., van Heerwaarden, J.S.: Adaptive dynamics, a geometrical study of the consequences of nearly faithful reproduction. In: van Strien, S.J., Verduyn Lunel, S.M. (eds.) Stochastic and spatial structures of dynamical systems, North-Holland, Amsterdam, 1996, pp 183-231

Kooijman, S.A.L.M.: Dynamic energy and mass budgets in biological systems. Cambridge University Press, Cambridge, 2000

Polis, G.A.: The evolution and dynamics of intraspecific predation. Ann. Rev. Ecol. Syst. 12, 225-51 (1981)

Van den Bosch, F., de Roos, A.M., Gabriel., W.: Cannibalism as a life boat meachanism. J. Math. Biol. 26, 619-633 (1988)

Van den Bosch, Gabriel., W.: Cannibalism in age structured populations. Theor. Popul. Biol. 59, 551-573 (1997) 